\title{
Tribology of silica nanoparticle-reinforced, hydrophobic sol-gel composite coatings
}

\author{
Derrick A. Banerjee, Aaron J. Kessman, Darran R. Cairns, Konstantinos A. Sierros* \\ West Virginia University, Dept. of Mechanical and Aerospace Engineering, Morgantown, WV 26506, USA \\ *Corresponding author. Tel.: +1 304290 7375. E-mail address: Kostas.Sierros@mail.wvu.edu
}

\begin{abstract}
Hydrophobically functional coatings can be used to protect surfaces and therefore improve the performance and lifetime of a broad range of applications such as optoelectronics and touchscreens. Organic-inorganic hybrid materials such as silica sol-gel coatings are particularly effective for this purpose, but the functional molecules in these coatings are susceptible to abrasive wear and thus lose their performance over time in the harsh environments typically encountered. To combat these problems, a silica nanoparticle-reinforced matrix was developed to increase hardness and wear resistance of the overall coating.
\end{abstract}

This study involved the abrasive wear analysis of fluorinated composite silica particle reinforced sol-gel silica coatings dip-coated on glass substrates. Varying amounts of silica nanoparticles from 0.5 to $10 \mathrm{wt} \%$ of the precursor weight were added to examine the structural dependence of abrasive wear mechanisms to elucidate strengthening mechanisms that could lead to improvements of coating properties. Abrasion was conducted using an in-house built reciprocating polishing wear apparatus. Characterization of the water contact angle of the coating was conducted to determine the hydrophobic functionality after wear cycles. Atomic force microscopy, lateral force microscopy, nanoindentation, nano-scratch, contact angle goniometry, and optical microscopy were performed at intervals of abrasive wear testing to characterize these wear mechanisms and the functional degradation of the coating. It was generally found that, among other possible factors, the increased indentation hardness of coatings led to a 
decreased wear rate. Additionally, an optimal amount of added colloidal silica of 1-2 wt\% of the precursors provided the best overall mechanical, tribological, and functional performance.

Keywords: hydrophobic coatings, sol-gel, colloidal silica, abrasive wear 


\section{Introduction}

Transparent, non-wetting coatings are useful for a wide range of surface-protective applications such as solar panels [1,2], information displays [3], touchscreens, ophthalmic lenses [4], optical storage disks [5], painting glazing [6], industrial, automotive, textile, household applications [7], architectural structures, and urban infrastructure [8]. Incorporation of wear-resistant hydrophobic layers can reduce waste and cleaning needs and extend the lifetime of these applications. Non-wetting coatings are most often based on materials with low mechanical strength and poor tribological performance. However, for many of these applications, resistance to abrasive wear is also important.

Conventional low-surface energy coatings such as polytetrafluoroethylene (PTFE) have poor mechanical strength which leaves them susceptible to abrasive wear [9]. An approach taken to offset these weaknesses is using a sol-gel method to apply a fluorinated organic monolayer [10]. This process provides a strong $\mathrm{Si}-\mathrm{O}$ film-substrate bond due to the sol-gel grafting [11] as well as high thermal stability, optical transparency, and substrate compatibility [12]. However, the setback for this approach is that these fluorinated moieties are thermodynamically driven to the solid-vapor interface during deposition $[13,14]$, leading to surface stratification and poor functionalization of the bulk coating. The top surface provides excellent functionality, but this molecularly thin monolayer also has an inherently low abrasive resistance.

An approach taken by Kessman et al. [11,15] uses a sol-gel process of co-condensation of silicon alkoxides and fluorosilanes. This process overcomes the surface stratification problem by encapsulating the functional moieties in mesopores within the bulk of the sol-gel matrix. While this provides a vast improvement for many applications, the induced porosity from this approach has a detrimental effect on the overall tribological performance of the coating. There was, however, a decrease in internal friction coefficient reported due to this encapsulation of fluorinated molecules, which in this case served to counteract the loss in wear resistance due to increased porosity.

It has also been shown that ceramic colloidal additives can be used in self-assembly processes to potentially improve overall film durability. Suegama et al. found an increase in the mean nanohardness value of sol-gel coatings by the addition of silica nanoparticles [16]. Lakshmi et al. showed that the 
addition of colloidal nanoparticles could also have the benefit of imparting a textural advantage to enhance the surface functional properties through increased roughness while simultaneously improving the pencil hardness parameter of the coating [17]. Hwang et al. reported a similar increase in hydrophobicity, but found a decrease in wear resistance with a macroscale abrasion test [18]. Each of these studies involved the agglomeration of colloidal nanoparticles at the surface of the coating increasing the macroscopic hardness and water contact angle of the coatings, but after abrasive wear the functionality still decreased significantly. The wear mechanisms and rates for this approach have not been adequately quantified. Also, the overall usefulness of this method is limited in its application since a specific substrate condition or texturing of the surface may undesirably affect the other properties of the coating. Furthermore, flocculation of the colloidal particles could also negatively affect the optical transmission of the coatings.

The realistic goal is to optimize the coatings such that they have the highest hardness and best wear resistance possible without compromising the functional properties. The focus of the work presented here is to further investigate the use of hard colloidal silica nanoparticles in sol-gel derived coatings and their distribution throughout the bulk of the coating. The effects of this addition need to be more precisely examined to determine the actual interplay between coating roughness, hardness, friction, and wear characteristics. Sol-gel derived silica coatings were deposited on glass substrates with varying amounts of added colloidal silica. The coatings were tested using a reciprocating wear apparatus and wear rates were calculated by measured film thickness after intervals of wear. Instrumented nanoindentation, nano-scratch testing, stylus profilometry, atomic force microscopy (AFM), and lateral force microscopy (LFM) were used to examine the effect of the colloidal silica on the characteristics of the coatings. Contact angle goniometry was also used to quantify the hydrophobicity of the coatings throughout the wear testing.

\section{Experimental procedure}

\subsection{Synthesis of films}

The synthesis of the silica nanoparticle-reinforced fluorinated coatings first involves the cocondensation of fluorinated silanes with an alkoxide silica precursor at room temperature $[11,15]$. The 
alkoxide precursor used was tetraethoxysilane (TEOS, tetraethyl orthosilicate 99\%, Acros Organics), and the functional fluorinated silane used was a perfluoropolyether silane (PFPE, Fluorolink S10, Solvay Solexis). The added colloidal silica nanoparticles used were $20 \mathrm{~nm}$ in diameter dispersed in ethylene glycol at a concentration of 30\% (DP5820, Nyacol Nano Technologies).

The sol-gel solutions were synthesized in approximately 25-30 g batches in the following order and in the ratios described. First, TEOS and PFPE were hydrolyzed with water, isopropyl alcohol (IPA), and a small amount of hydrochloric acid $(\mathrm{HCl})$ in the molar ratio 0.98 TEOS : 0.02 PFPE : 4 H2O : 1 IPA : $0.01 \mathrm{HCl}$ and stirred for 1 hour using a magnetic stirrer. The sol was then diluted with IPA and butanol $(\mathrm{BtOH})$ for a final molar ratio of 0.98 TEOS : 0.02 PFPE : $4 \mathrm{H} 2 \mathrm{O}: 10 \mathrm{IPA}: 0.01 \mathrm{HCl}: 2 \mathrm{BtOH}$. The next step was to add the colloidal silica at varying concentrations. These silica concentrations were calculated as a percent of the combined weight of precursor TEOS and PFPE used and varied from 0 to 10 weight percent. After mixing for another 10 minutes, the sols were dip coated onto soda-lime glass microscope slides using a KSV Instruments dip coater with a withdrawal speed of $200 \mathrm{~mm} / \mathrm{min}$. All coatings were synthesized and deposited in controlled ambient conditions of $23 \pm 1^{\circ} \mathrm{C}$ and $40 \pm 10 \%$ relative humidity. After deposition, the films were dried for 24 hours at ambient conditions. Finally, the coatings were cured in a box furnace at $200^{\circ} \mathrm{C}$ for 2 hours with a ramp rate of $10^{\circ} \mathrm{C} / \mathrm{min}$ from ambient conditions.

\subsection{Material characterization}

In order to determine the coatings' functional sustainability and resistance to abrasive wear, they were worn using a lab-built reciprocating polishing device. This instrument has been used previously for wear studies on sol-gel coatings $[11,15,19]$. The device provides a conformal surface that uniformly and gradually wears through coatings with thicknesses on the order of several hundred nanometers in a controlled manner. The instrument consists of a vertically-loaded reciprocating polishing contact controlled by a stepper motor (NM34A200, Zaber Technologies). The coating abrasion occurs from trapped $0.3 \mu \mathrm{m}$ alumina particles (90-187120, Allied High Tech Products) in a cloth pad (90-150285, Allied). The alumina particles are suspended in an aqueous slurry that is regularly replenished, and the cloth pad is rotated after wear intervals to give a pristine surface for abrasion. The normal load was kept 
constant at $4.70 \mathrm{~N}$. The polishing contact was determined to be over a nominal area of $175 \mathrm{~mm}^{2}$ for an approximate Hertzian contact pressure of $27 \mathrm{kPa}$. The stroke length of the reciprocating contact was 35 $\mathrm{mm}$ at $0.5 \mathrm{~Hz}$. These parameters were chosen to model contact wear on a touchscreen or cleaning of solar panels. Samples were removed at regular intervals for analysis and cleaned with water and alcohol to remove residue from abrasion.

Hydrophobicity was quantified using a lab-built sessile drop contact angle goniometer. Five approximately $5-\mu \mathrm{L}$ droplets of deionized water were placed over a macroscopic area of the coating approximately 1-2 mm apart using a manual microsyringe. Images of the horizontal view of the drops were captured using a digital microscope and analyzed using the low-bond axisymmetric drop shape analysis (L-BADSA) method in the "Drop Shape Analysis" plugin [20] for ImageJ. Optical transmission of the coatings was measured using a UV-Vis spectrometer (Jaz Spectrometer Module, Ocean Optics) with the deuterium-tungsten halogen light source module.

Coating thickness and roughness was measured on a Veeco Dektak 150 (Bruker AXS) stylus profilometer by measuring the depths of manual scratches through the center of the coatings. The Dektak 150 stylus used had a $12.5 \mu \mathrm{m}$ radius tip with a nominal applied normal force of $0.1 \mathrm{mN}$. Scan lengths used for roughness measurements were between 500 and $1000 \mu \mathrm{m}$. Film wear rates were calculated from a weighted linear least squares fit of film thickness versus reciprocating wear cycles.

AFM and LFM provided high resolution surface and friction mapping of the coatings. AFM and LFM were performed using a Molecular Imaging PicoScan 3000 system in contact mode. A silicon tip (SC37B, MikroMasch) on a cantilever with force constant $\mathrm{k}=0.3 \mathrm{~N} / \mathrm{m}$ and radius of curvature less than $10 \mathrm{~nm}$. Deflection and force were calibrated by using the slope of the force curve on the approach to the sample surface and the nominal cantilever spring constant. Relative friction measurements were made by applying forces in the range of 5-50 $\mathrm{nN}$ to the tip, scanning over a $500 \mathrm{~nm}$ by $500 \mathrm{~nm}$ area at a speed of $10 \mu \mathrm{m} / \mathrm{s}$. All measurements were conducted in controlled ambient conditions of $22 \pm 1^{\circ} \mathrm{C}$ and $40 \pm 10 \%$ relative humidity.

A nanoindentation system (NHT, CSM Instruments) and an ultra-nanoindentation system (UNHT, CSM Instruments) were used to determine the instrumented hardness values of the coatings. A standard 
indentation method was used for each with a 30 second load time, followed by a 10 second pause, and a 30 second unload. The maximum penetration depth into the coatings was kept less than $10 \%$ of the coating thickness to minimize substrate effects on the hardness values. This corresponded to a maximum normal load of $0.5-0.75 \mathrm{mN}$. A Berkovich geometry diamond tip was used with both the NHT and UNHT, and a $10 \mu \mathrm{m}$ radius sphero-conical diamond tip was also used on the NHT.

To determine the coating adhesion characteristics and the normal load at which critical failure of the coatings occurs, scratch testing was performed using a Nano-Scratch Tester (NST, CSM Instruments). Samples were mounted on a standard resolution friction table (ST-142, $0.01 \mathrm{mN}$ friction force resolution) and scratched with a $10 \mu \mathrm{m}$ radius Rockwell diamond tip indenter. A progressively increasing load scratch program was used from $5 \mathrm{mN}$ to $75 \mathrm{mN}$ over a length of $500 \mu \mathrm{m}$, with a loading rate of 140 $\mathrm{mN} / \mathrm{min}$. Critical failure loads were determined from depth and friction feedback as well as panoramic images of the scratches.

\section{Results and Discussion}

The functional sustainability after intervals of abrasive wear cycles for each coating with added colloidal silica is shown in Fig. 1. The coatings tested had 0, 0.5, 1, 2, 5, and $10 \mathrm{wt} \%$ added colloidal silica. The thickness of the coatings ranged between 500 and $700 \mathrm{~nm}$ with the larger thicknesses generally corresponding to higher concentrations of colloidal silica. Coating thickness was measured after intervals of wear cycles and normalized based on the initial thickness for comparison purposes. The optical transmission across the visible range was between 90 and $92 \%$ for coatings up to and including 5 wt $\%$ added colloidal silica. The transmission for $10 \mathrm{wt} \%$ coatings varied between 75 and $80 \%$.

For all coatings tested except the $10 \mathrm{wt} \%$ added silica, the initial water contact angle was above $95^{\circ}$, all with standard deviations of less than $3^{\circ}$. The $10 \mathrm{wt} \%$ added colloidal silica had an initial contact angle consistently of $85-90^{\circ}$. After the initial interval of wear of a total sliding distance of $7 \mathrm{~m}$, these contact angles decreased by between $10^{\circ}$ and $30^{\circ}$ from the value of the pristine coating. However, the contact angles did not decrease significantly after that point as shown by the nearly flat curves after $20 \%$ normalized depth into the coating. There is a distinct decline in hydrophobicity for coatings with no 
added colloidal silica (shown as $0 \%$ ), which has been attributed to the surface stratification of the fluorinated silanes in previous studies [15]. However, as colloidal silica is added to reinforce the matrix, there was an apparent effect on the functional sustainability of the coatings. This change can possibly be attributed to better dispersion or encapsulation of the fluorosilanes into the bulk of the coating due to the added colloidal silica. While this phenomenon may be a product of multiple factors, it has been noted previously [16] that there is a noticeable induced porosity from added organic molecules within a sol-gel matrix, causing the fluorosilanes to migrate to this solid-vapor interface thereby increasing the bulk hydrophobicity. In this case, the added ethylene glycol/colloidal silica solution may cause this increased porosity.

The average roughness values of the coatings were found to increase slightly with the increase of concentration of colloidal silica, as measured with the stylus profilometer. Up to a loading of $2 \mathrm{wt} \%$, the $\mathrm{R}_{\mathrm{a}}$ values were generally below $5 \mathrm{~nm}$, with this value increasing to between $10-15 \mathrm{~nm}$ for 5 and $10 \mathrm{wt} \%$ coatings. After any number of abrasive wear cycles, the $\mathrm{R}_{\mathrm{a}}$ increased to between 10 and $20 \mathrm{~nm}$ for coatings with $2 \mathrm{wt} \%$ or less added colloidal silica. For coatings with $5 \mathrm{wt} \%$ or more added colloidal silica, this $\mathrm{R}_{\mathrm{a}}$ increased further to between 20 and $25 \mathrm{~nm}$. This distinct separation in measured roughness values is likely attributed to a larger particle size on the surface due to agglomeration of the silica nanoparticles in the sol and surface segregation [21]. Then after the coating is worn, these portions become exposed.

Averaged over the wear cycles, the contact angle is lowest at $10 \mathrm{wt} \%$ and highest at $2 \mathrm{wt} \%$ for coatings with added colloidal silica. It can be concluded that increasing the concentration of added colloidal silica beyond $2 \mathrm{wt} \%$ has a negative effect on the functional durability. This implies that there could be an optimal amount of added porosity, increased surface roughness, and beyond that point it becomes detrimental to the functional performance of the coatings.

Fig. 2 shows the hardness values found from nanoindentation plotted with the wear rates as a function of the concentration of added colloidal silica. As expected, this graph shows an inverse relationship between the indentation hardness and the wear rate. Thus with improved hardness, the abrasive wear resistance of the coatings increases. The highest hardness values measured were for 1 and 2 
wt $\%$ added colloidal silica. The mean hardness increased from $1.38 \mathrm{GPa}$ at $0 \mathrm{wt} \%$ to $2.8 \mathrm{GPa}$ at $10 \mathrm{wt} \%$. For comparison purposes, the bare glass slides used were also tested and the mean hardness value was found to be about 7.5 GPa.

The concentrations with the highest hardness corresponded to the lowest wear rates as calculated by thickness measurements and the Archard equation [22]. The hardness values doubled and the wear rate decreased by over $35 \%$ from 0 to $2 \mathrm{wt} \%$. After this point, however, the hardness values decreased slightly. This can be attributed to the increased porosity and roughness which can greatly affect instrumented indentation results depending on where the indenter tip contacts the sample surface. This also led to a significantly higher standard deviation for indentation results.

It might be expected that the wear rate be more linearly related to the hardness value of the coating, but the relationship between the friction and wear mechanisms must also be considered. To analyze the wear mechanisms and the subsurface properties of the coatings, optical microscopy, AFM, and LFM were used. Fig. 3 shows optical micrographs of a 2 wt $\%$ coating (a) before and (b) after wear cycles. Fig. 3a clearly shows the aforementioned surface agglomeration of the colloidal silica, with this becoming more well-defined with higher concentrations of added silica. After 100 wear cycles, these flocs are predictably worn in a pattern parallel to the sliding direction. As expected from previous studies, the coatings wear gradually due to cohesive failure, with no delamination or scratches penetrating to the substrate. The coating does wear less uniformly than expected, likely due to the increased initial surface roughness. The surface also appears to be rougher after wear, as was also found from profilometry. These wear patterns were similar for all concentrations, thus the added silica did not have a noticeable effect on the wear mechanisms at this scale.

With the AFM analysis of the same coatings in Fig. 4, similar kinds of surface properties and wear characteristics as with the optical microscopy are seen. This figure shows the deflection of the AFM tip on a $2 \mathrm{wt} \%$ added colloidal silica coating (a) before and (b) after 100 wear cycles. The coating's microstructure appears to include smaller flocs within the matrix of larger particles agglomerated on the surface of the coating. The top surface of the coating is worn away similarly to the larger scale optical 
micrographs, but on this smaller scale there are signs of abrasive failure as the larger flocs on the surface are removed. These can be seen as the darker spots in Fig. $4 \mathrm{~b}$.

To further understand the relationship between wear and friction characteristics, LFM was performed on the coatings. Fig. 5 shows representative LFM micrographs on a $5 \mathrm{wt} \%$ added colloidal silica coating (a) before and (b) after 100 wear cycles. These figures show lower friction surfaces with darker shades and higher friction surfaces with lighter shades. As expected, the surfaces with the highest friction forces correspond to the rough flocs on the pristine surface and the remains of these flocs after wear cycles. The lower friction signals correspond to the sol-gel matrix material. To quantify the differences in these characteristics, the friction force vs. normal force relationship was found for all of the coatings through applying increasing voltages to the AFM tip. This is shown in Fig. 6. It was generally found that the worn coatings had a higher average frictional signal than the unworn coatings for each added colloidal silica concentration. Also, generally the frictional signal increased with increased colloidal silica concentration. They are also largely a function of the increased roughness from the increasing concentrations of added colloidal silica.

While this higher friction has been shown to lead to higher wear rates in previous studies of similar coatings $[19,23]$, the higher hardness coupled with some encapsulation of fluorinated silanes within the pores of the bulk of the coating serves to not only mitigate but to overcome this expected increased wear rate. The slightly higher frictional forces seem to only lessen the overall effectiveness of the matrix-reinforcement. This follows the previous results of the characterization of wear rate and hardness.

Nano-scratch testing was used to further investigate the adhesion characteristics of the coatings to the glass substrates. Fig. 7 shows panoramic optical micrographs of representative scratches on coatings (a) without added colloidal silica and (b) with $2 \mathrm{wt} \%$ added colloidal silica. The linearly progressive normal loading increases from left to right of these images. The critical loads are marked $\mathrm{L}_{\mathrm{C} 1}$ and $\mathrm{L}_{\mathrm{C} 2}$ for both samples on Fig. 7 with red arrows. For these coatings, these critical loads signify the normal load at which the coatings begin to crack and at which spallation or delamination occurs, respectively. The slight 
plastic deformation from ploughing into the surface of Fig. $7 b$ before $\mathrm{L}_{\mathrm{C} 1}$ is not considered a critical load as it was not consistently observed on these coatings.

For the coating without added silica, the $\mathrm{L}_{\mathrm{C} 1}$ value is $19 \pm 1 \mathrm{mN}$; for the coating with $2 \mathrm{wt} \%$ added colloidal silica, the first sign of cracking occurs at $27 \pm 3 \mathrm{mN}$. The $\mathrm{L}_{\mathrm{C} 2}$ values are consistently $36 \pm 3 \mathrm{mN}$ and $44 \pm 3.5 \mathrm{mN}$ for 0 and $2 \mathrm{wt} \%$ coatings, respectively. This is considered the point of catastrophic failure of the coatings. The failure mode between $\mathrm{L}_{\mathrm{C} 1}$ and $\mathrm{L}_{\mathrm{C} 2}$ for both of the coatings can be described as a cohesive failure with plastic deformation and some slight spallation. At the $\mathrm{L}_{\mathrm{C} 2}$ normal load, shown more closely in Fig. 8, both coatings begin to delaminate and spall more easily than before. However, the wear tracks are wider for the $2 \mathrm{wt} \%$ coating than the coating without added colloidal silica. There are also larger debris particles present in the $2 \mathrm{wt} \%$, indicating a more adhesive failure. Overall these nano-scratch results indicate a better initial adhesion strength and wear resistance for the coating with added colloidal silica than without based on the normal loads at which the respective coatings begin to crack and delaminate. However, the failure mechanisms present in the $2 \mathrm{wt} \%$ coating indicate that beyond the surface of the coating the adhesion strength could be negatively affected by the addition of the colloidal silica and the resulting induced porosity or change in microstructure.

\section{Conclusions}

Sol-gel derived fluorinated coatings with added colloidal silica were deposited on glass substrates. Their mechanical, tribological, and functional properties were then analyzed to elucidate the effect of varying concentrations of colloidal silica on the performance of the coatings. The overall goal of this research study was to synthesize more wear-resistant sol-gel coatings that were hydrophobic, thin, thermally stable, transparent, and easily deposited.

The instrumented indentation hardness of the coatings increased as expected with increased concentration of colloidal silica up to and including $2 \mathrm{wt} \%$. It is likely that the nanoparticles (average diameter of $20 \mathrm{~nm}$ in suspension) aggregated and surface segregated at these higher concentrations, and this is further confirmed through abrasive wear testing and atomic and lateral force microcopy. The corresponding wear rates were lower as expected with increased hardness up to this same $2 \mathrm{wt} \%$ critical 
point. The hydrophobicity of the coatings was also increased through the addition of colloidal silica nanoparticles. This was concluded to be a result of the encapsulation of the fluorinated silanes within the bulk of the coating due to an induced porosity from the colloidal silica. The highest functional sustainability averaged throughout the wear of the coatings was achieved at 1 and $2 \mathrm{wt} \%$ added colloidal silica. Thus, it can be concluded that the concentration of fluorosilanes was sufficiently high enough to be encapsulated within the matrix of the coating. The water contact angles for these coatings between 0.5 and $5 \mathrm{wt} \%$ were consistently between $70^{\circ}$ and $90^{\circ}$ after any number of wear cycles, compared to around $50^{\circ}$ for coatings without colloidal silica. The addition of colloidal silica to the sol-gel formulation also generally increased the critical load of adhesive failure through nano-scratch studies. The additional colloidal silica also led to a more destructive delamination wear mechanism.

Overall, increasing concentrations of added colloidal silica to the sol-gel matrix led to a harder and more wear-resistant coating on the surface and within the subsurface, while also inducing some adverse effects from an inferred increase in coating porosity. To an extent, this reduced the wear-resistant effects of the increased hardness of the surface. However, the mechanical reinforcement and some encapsulation of fluorinated molecules within the bulk of the coating leading to an improved functional performance is a net-gain compared to coatings without the added colloidal silica.

\section{Acknowledgements}

DAB acknowledges the WVU Shared Research Facilities and the Ruby Doctoral Fellowship. AJK acknowledges the support of the Department of Defense (DoD) through the National Defense Science \& Engineering Graduate Fellowship (NDSEG) Program and a Graduate Research Fellowship from the National Science Foundation (NSF-GRFP). DRC and AJK acknowledge the support of the WVU EPSCoR. The authors would like to give special thanks to Edward Chambers, CSM Instruments, and the nCATS lab group at the University of Southampton for the use of their time and equipment. 


\section{References}

[1] H. Elminir, A. Ghitas, R. Hamid, F. El-Hussainy, M. Beheary, K. Abdel-Moneim, Effect of dust on the transparent cover of solar collectors, Energ. Convers. Manag. 47 (18-19) (2006) 3192-3203.

[2] P. Hinz, H. Dislich, Anti-reflecting light-scattering coatings via the sol-gel-procedure, J. Non-Cryst. Solids 82 (1-3) (1986) 411-416.

[3] C. Schelle, M. Mennig, H. Krug, G. Jonschker, H. Schmidt, One step antiglare sol-gel coating for screens by sol-gel techniques, J. Non-Cryst. Solids 218 (1997) 163-168.

[4] F. Samson, Ophthalmic lens coatings, Surf. Coat. Tech. 81 (1) (1996) 79-86.

[5] G. Wu, J. Wang, J. Shen, T. Yang, Q. Zhang, B. Zhou, Z. Deng, F. Bin, D. Zhou, F. Zhang, Properties of sol-gel derived scratch-resistant nano-porous silica films by a mixed atmosphere treatment J. NonCryst. Solids 275 (2000) 169-174.

[6] A. Vilcnik, I. Jerman, A. Vuk, M. Kozelj, B. Orel, B. Tomsic, B. Simonic, J. Kovac, Structural properties and antibacterial effects of hydrophobic and oleophobic sol-gel coatings for cotton fabrics Langmuir 25 (10) (2009) 5869-5880.

[7] K. Johns, G. Stead, Fluoroproducts - the extremophiles, J. Fluorine Chem. 104 (1) (2000) 5-18.

[8] A. Cannavale, F. Fiorito, M. Manca, G. Tortorici, R. Cingolani, G. Gigli, Build. Environ. 45 (2010) 1233-1243.

[9] K. Tanaka, Y. Uchiyama, S. Toyooka, The mechanism of wear of polytetrafluoroethylene, Wear 23 (1973) 153-172.

[10] T. Yoneda, T. Morimoto, Mechanical durability of water repellent glass, Thin Solid Films 351 (1-2) (1999) 279-283.

[11] A. Kessman, E. DeFusco, A. Hoover, K. Sierros, D. Cairns, Structural, mechanical, and tribological properties of fluorinated mesoporous silica films: Effect of functional moiety and surfactant template concentrations, Thin Solid Films 520 (2012) 3896-3903.

[12] J. Mackenzie, E. Bescher, Physical properties of sol-gel coatings, J. of Sol-Gel Sci. Technol. 19 (1) (2000) 23-29.

[13] M. Toselli, J. Gardella, M. Messori, A. Hawkridge, F. Pilati, C. Tonelli, Surface chemical analysis of polyepsilon-caprolactone-perfluoropolyether-polyepsilon-caprolactone triblock copolymers, Polym. Int. 52 (8) (2003) 1262-1274.

[14] P. Fabbri, M. Messori, M. Montecchi, S. Nannarone, L. Pasquali, F. Pilati, C. Tonelli, M. Toselli, Perfluoropolyether-based organic-inorganic hybrid coatings, Polymer 47 (4) (2006) 1055-1062.

[15] A. Kessman, D. Cairns, Template-assisted encapsulation of fluorinated silanes in silica films for sustained hydrophobic-oleophobic functionality, J. Colloid Interf. Sci. 360 (2) (2011) 785-792.

[16] P. Suegama, A. Recco, A. Tschiptschin, I. Aoki, Influence of silica nanoparticles added to an organosilane film on carbon steel electrochemical and tribological behavior, Prog. Org. Coat. 60 (2) (2007) 90-98.

[17] R. Lakshmi, T. Bharathidasan, B. Basu, Superhydrophobic sol-gel nanocomposite coatings with enhanced hardness, Appl. Surf. Sci. 257 (24) (2011) 10421-10426. 
[18] J.H. Hwang, B. Lee, V. Klep, I. Luzinov, Transparent hydrophobic organic-inorganic nanocomposite films, Mater. Res. Bull. 43 (2008) 2652-2657.

[19] A. Kessman, S. Kukureka, D. Cairns, Tribology of non-wetting fluorinated mesoporous silica films, Wear 271 (9-10) (2011) 2144-2149.

[20] A. Stalder, T. Melchior, M. Müller, D. Sage, T. Blu, M. Unser, Low-bond axisymmetric drop shape analysis for surface tension and contact angle measurements of sessile drops, Colloids Surf., A 364 (1-3) (2010) 72-81.

[21] A. Kessman, D. Cairns, P. Richter, F. Bottari, Mesostructured island formation in sol-gel SiO2 films through controlled, concentration-dependent flocculation of colloidal silica particles, Mater. Lett. 64 (2010) 258-260.

[22] E. Rabinowicz, L. Dunn, P. Russell, A study of abrasive wear under three-body conditions, Wear 4 (5) (1961) 345-371.

[23] A. Kessman, D. Cairns, Microtribological Study of Internal Surfaces of Fluorinated Mesoporous Silica Films, Langmuir 27 (2011) 5968-5975. 


\section{List of Figure Captions}

Fig. 1: Water contact angle of coatings as a function of the penetration depth into the coating as a percent of the initial film thickness after intervals of abrasive wear cycles. Data are shown for coatings with varying concentrations of added colloidal silica.

Fig. 1: Instrumented nanoindentation hardness and wear rates of coatings with varying amounts of added colloidal silica.

Fig. 3: Representative optical micrographs of (a) unworn and (b) partially worn coatings with 2 wt\% added colloidal silica. Sliding direction is up and down as shown for worn sample.

Fig. 4: AFM contact mode micrographs imaged at $5 \mathrm{nN}$ normal force of (a) unworn (vertical topography range of $30 \mathrm{~nm}$ ) and (b) partially worn coatings with $2 \mathrm{wt} \%$ added colloidal silica (vertical topography range of $120 \mathrm{~nm}$ ). Sliding direction is left-right as shown for the worn sample.

Fig. 5: Lateral force microscopy friction micrographs imaged (a) at $20 \mathrm{nN}$ normal force on an unworn coating and (b) $5 \mathrm{nN}$ normal force on a partially worn coating, both with $5 \mathrm{wt} \%$ added colloidal silica. Sliding direction is left-right as shown for worn sample.

Fig. 6: Lateral force microscopy friction data of (a) unworn and (b) partially worn coatings with varying concentrations of added colloidal silica. The friction signal versus normal load curves are normalized to the friction coefficient of bare glass.

Fig. 7: Representative panoramic optical micrographs of progressive loading nano-scratch tests on coatings (a) without added colloidal silica and (b) with $2 \mathrm{wt} \%$ added colloidal silica. Arrows signify critical failure loads on each coating. Scratches were $500 \mu \mathrm{m}$ in length and from $5-75 \mathrm{mN}$ in normal load with a $10 \mu \mathrm{m}$ radius Rockwell diamond tip.

Fig. 8: Optical micrographs of second critical load $\left(\mathrm{L}_{\mathrm{C} 2}\right)$ from nano-scratch testing of coatings (a) without added silica and (b) with 2 wt $\%$ added colloidal silica. 


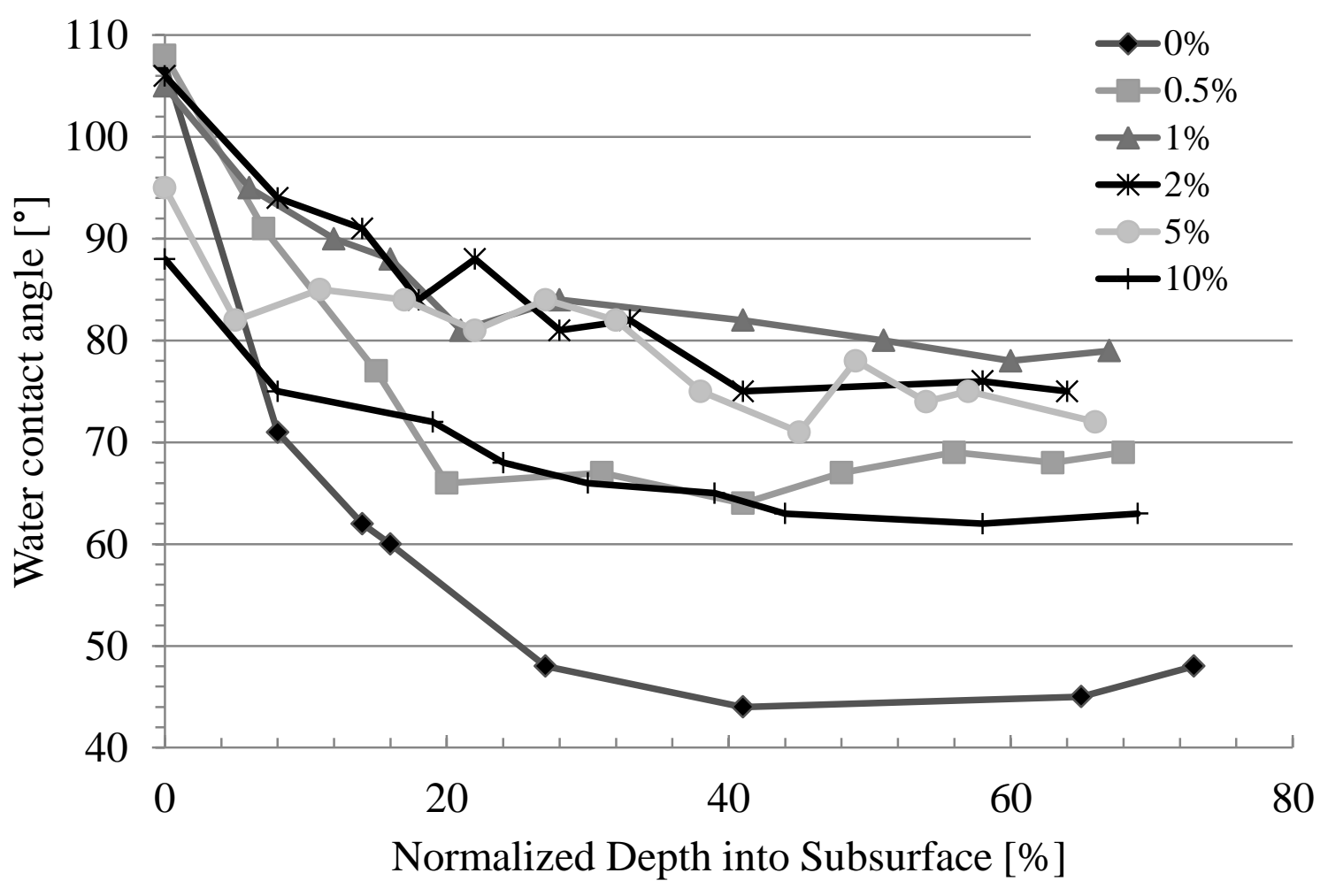

figure 1 




figure 2 



figure 3 

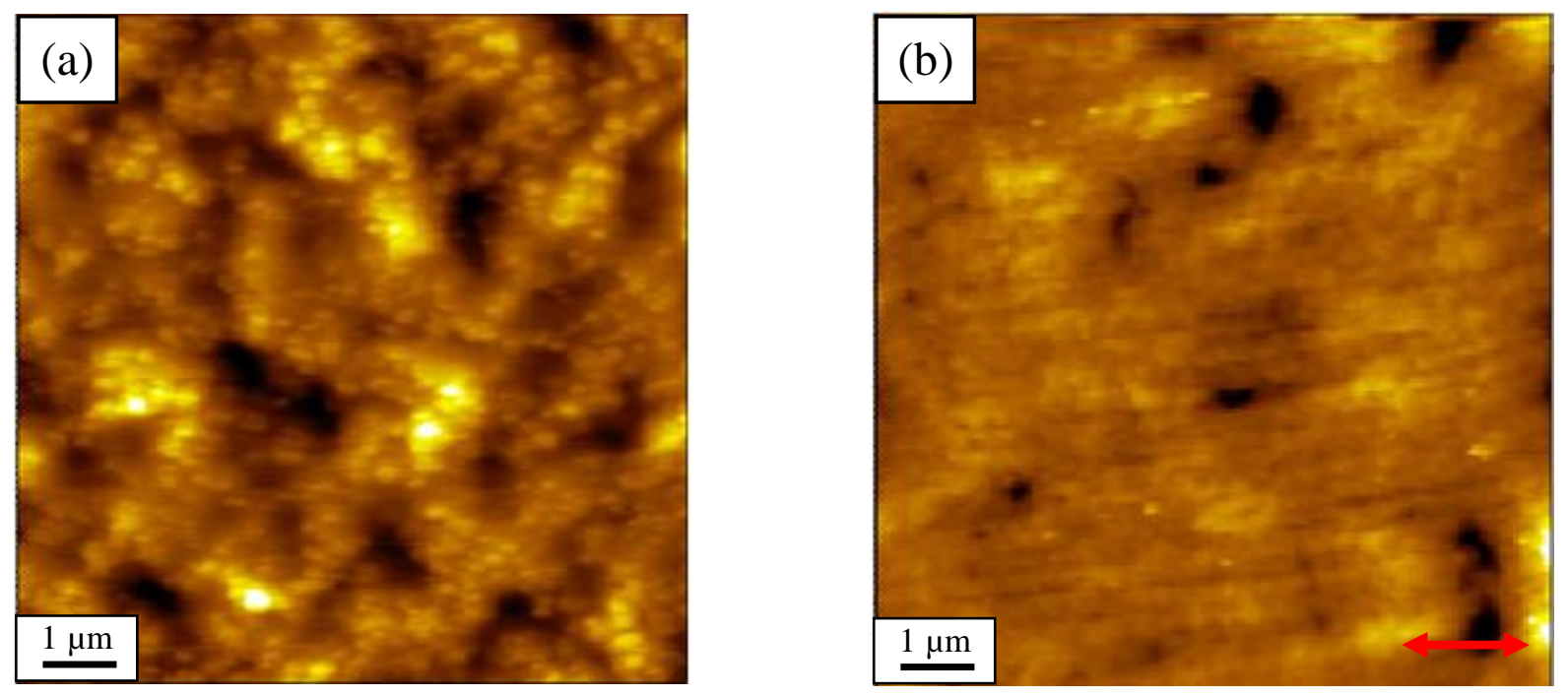

figure 4 

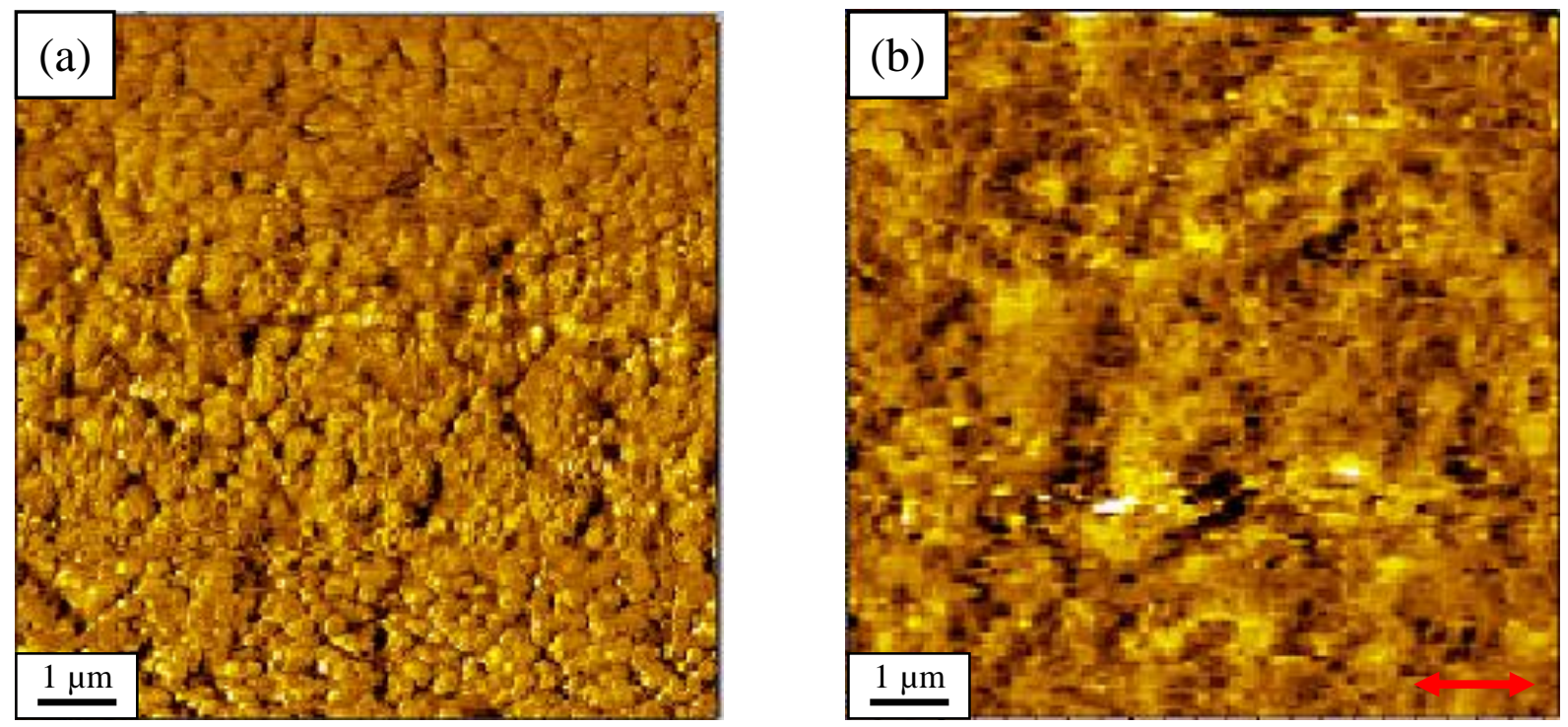

\section{figure 5}




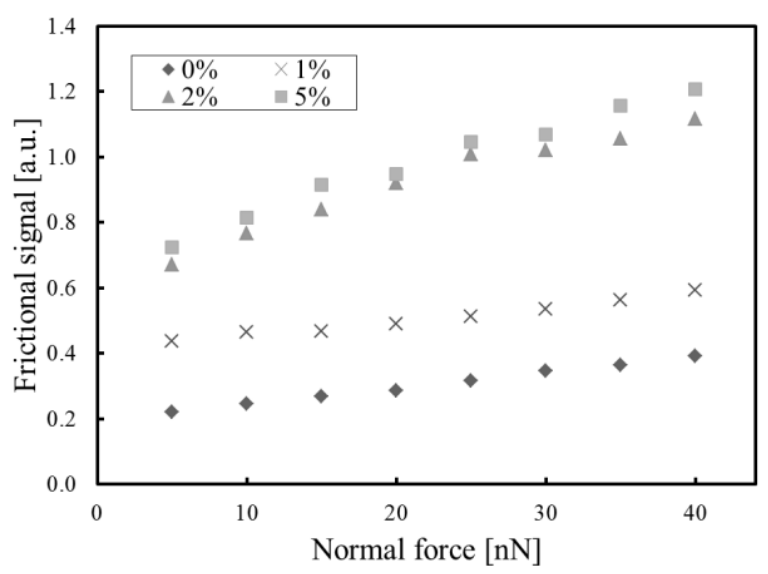

(a)

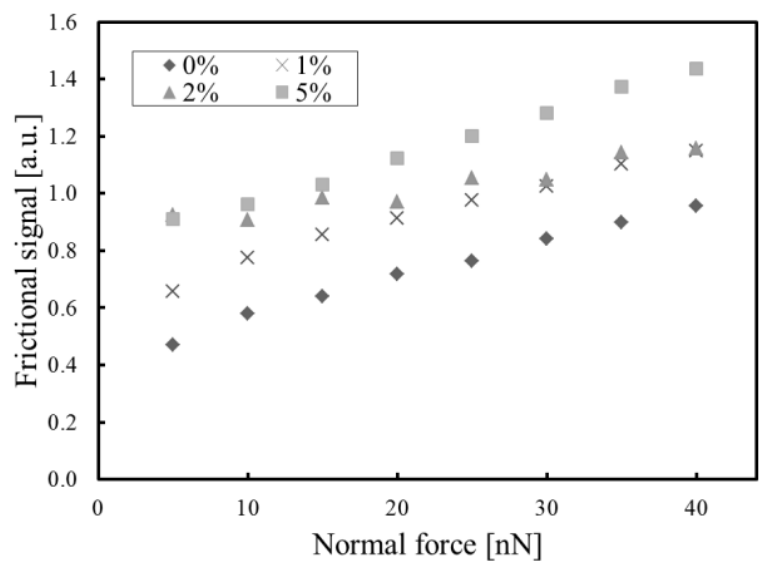

(b)

figure 6 


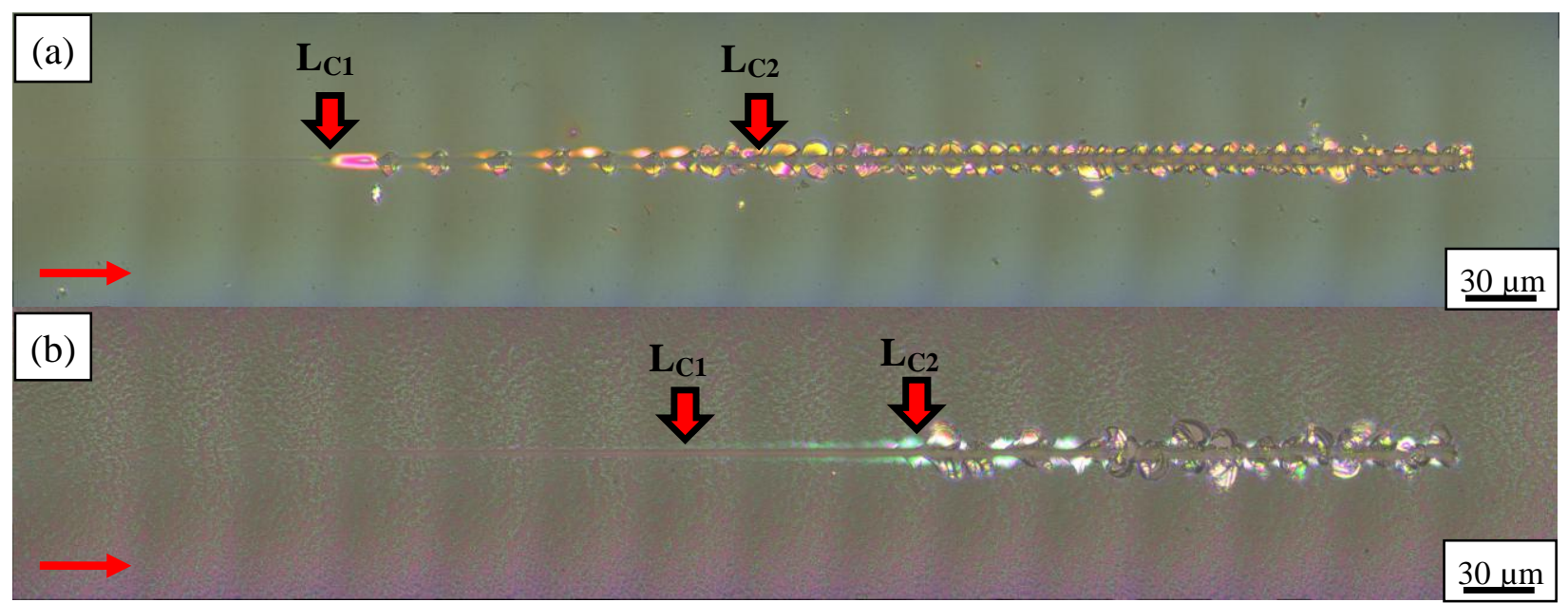

figure 7 


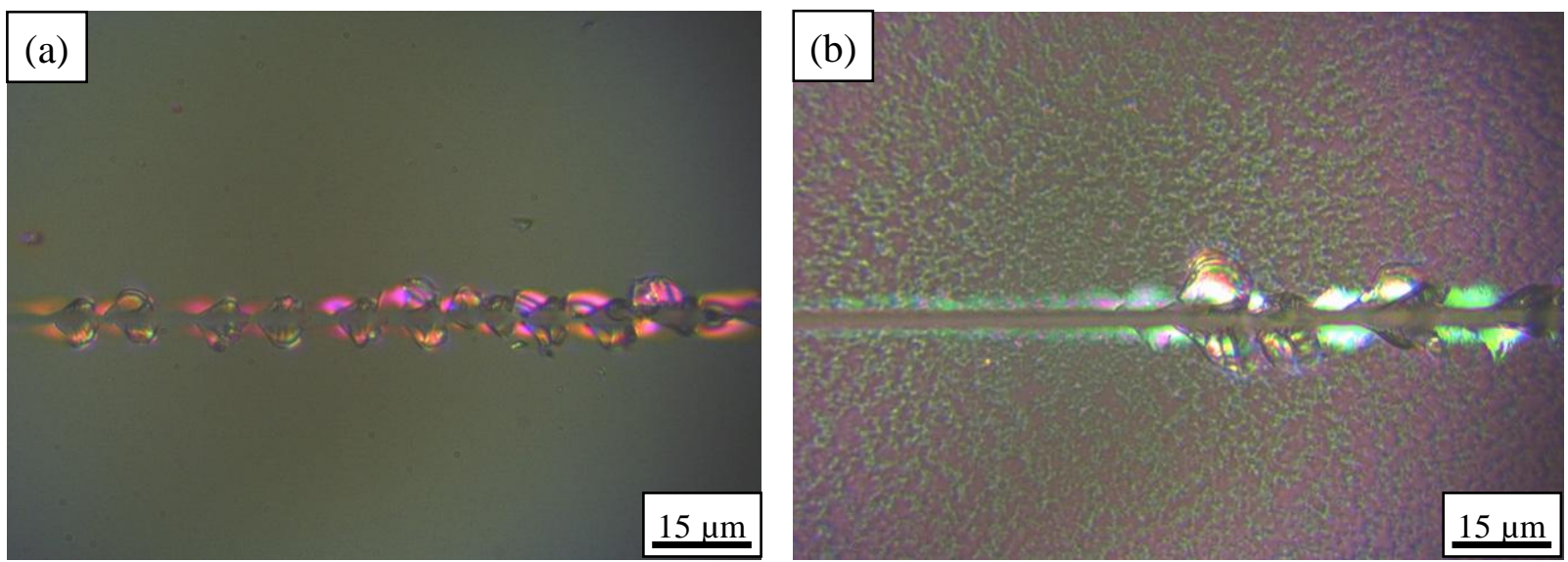

figure 8 